\title{
Emerging Challenges for Neuroradiologists
}

\author{
Horst Urbach ${ }^{1}$
}

Published online: 3 May 2017

(c) Springer-Verlag Berlin Heidelberg 2017

MRI and CT machines produce far more data than humans are able to process. Considering our epilepsy-dedicated MRI protocol, around $7 \times 106$ voxels are "filled up" within $35 \mathrm{~min}$, although for image interpretation, only some voxels are considered [1]. The voxel "load" is being steadily increased by accelerating image acquisition (e. g. with simultaneous multi-slice acquisitions, compressed sensing or statistical methods) and by post-processing algorithms generating additional parameter maps (consider, e.g., the various perfusion maps generated from a single dynamic susceptibility contrast [DSC] sequence). It is obvious that we will continue to need the support of computers to handle the huge amount of information (big data), to process and visualize all data (-omics), and also to compare results with comprehensive control databases. Luckily, we have computers and algorithms that are able to learn from former cases. In some applications, computer (machine) accuracy has already reached or even exceeded that of humans $[2,3]$ and machine learning will thus also displace much of the work (neuro-)radiologists used to do. Should we be worried about this? [4]. Not at all. We should take the chance and join with computer scientists. We are the ones to control the input data and define appropriate applications. Think also of the future: Algorithms reading cortical activity directly from the brain, transmitting signals from a paralyzed human's motor cortex to hand muscles and restoring motor control [5]. Or cortical activity down-regulated via "neuro-

Horst Urbach

horst.urbach@uniklinik-freiburg.de

1 Klinik für Neuroradiologie, Universitätsklinikum Freiburg, Freiburg, Germany feedback", which may be used to suppress tinnitus [6,7] or improve speech recovery after stroke. Such advances would unimaginable without machine learning and processing of real-time high-resolution physiological data. It is time to address the challenges and opportunities of machine learning.

This is one of the topics of the 52nd Annual Conference of the German Society of Neuroradiology (DGNR). Join us in Cologne (October 11-14th, 2017) and be inspired by the diversity of neuroradiology!

\section{References}

1. Urbach H, Mast H, Egger K, Mader I. Presurgical MR imaging in epilepsy. Clin Neuroradiol. 2015;25(Suppl 2):151-5.

2. Lao Z, Shen D, Xue Z, Karacali B, Resnick SM, Davatzikos C. Morphological classification of brains via high-dimensional shape transformations and machine learning methods. Neuroimage. 2004;21(1):46-57.

3. Esteva A, Kuprel B, Novoa RA, Ko J, Swetter SM, Blau HM, Thrun S. Dermatologist-level classification of skin cancer with deep neural networks. Nature. 2017;542(7639):115-8.

4. Obermeyer Z, Emanuel EJ. Predicting the future - big data, machine learning, and clinical medicine. N Engl J Med. 2016;375(13): 1216-9.

5. Bouton CE, Shaikhouni A, Annetta NV, Bockbrader MA, Friedenberg DA, Nielson DM, Sharma G, Sederberg PB, Glenn BC, Mysiw WJ, Morgan AG, Deogaonkar M, Rezai AR. Restoring cortical control of functional movement in a human with quadriplegia. Nature. 2016;533(7602):247-50.

6. Emmert K, Kopel R, Koush Y, Maire R, Senn P, Van De Ville D, Haller S. Continuous vs. intermittent neurofeedback to regulate auditory cortex activity of tinnitus patients using real-time fMRI - a pilot study. Neuroimage Clin. 2017;14:97-104.

7. Sitaram R, Ros T, Stoeckel L, Haller S, Scharnowski F, Lewis-Peacock J, Weiskopf N, Blefari ML, Rana M, Oblak E, Birbaumer N, Sulzer J. Closed-loop brain training: the science of neurofeedback. Nat Rev Neurosci. 2017;18(2):86-100. 